\title{
Information systems for urologists: the message from Mansoura, Egypt
}

\author{
Peter T Scardino and Paul Fearn
}

Relative to commercial enterprises, medicine has been slow to adopt computer information systems. Whereas systems for electronic billing and appointment scheduling are widely used, no institution has developed a satisfactory electronic medical record. Many medical centers collect and store electronic documents, spreadsheets or optical images, but none fully delivers to the practicing physician the right information, in the right format, at the right time. Why not? Surely the technology utilized in other sectors could be applied to medicine.

One key issue is the active participation of physicians in the design and development of systems that perform the functions we want, quickly and simply. Physicians worldwide have limited time to document their diagnostic and therapeutic activities, often delegating this to others.

When a data manager, or similar professional, abstracts structured data from transcribed notes, errors and biases can accumulate, and research based on these data may be skewed. Collecting duplicate data for clinical trials or outcomes research is labor intensive and expensive. If information for medical records, outcomes research and clinical trials could be gathered simultaneously, time and money could be saved. Several years ago, the development of a system to integrate research with clinical practice was initiated at our center; it has been a slow and expensive process.

I was surprised to learn recently that a successful clinical information system has been in regular use for years in Mansoura, Egypt. Last summer, we witnessed the system in action at the Mansoura Institute for Urology and Nephrology, a center of excellence that provides care to tens of thousands of patients, and education and training to hundreds of nurses and physicians from around the world. It works remarkably well, and is a
If information
for medical
records,
outcomes
research
and clinical
trials could
be gathered
simultaneously,

time and money could be saved.

PT Scardino is the Editor-in-Chief of Nature Clinical Practice Urology, Chairman of the Department of Urology at Sidney Kimmel Center for Prostate and Urologic Cancers, and Alfred P Sloan Chair at Memorial Sloan-Kettering Cancer Center. P Fearn is a Clinical Database Specialist at Memorial Sloan-Kettering Cancer Center.

\section{Competing interests}

The authors declared

they have no competing interests.

www.nature.com/clinicalpractice doi:10.1038/ncpuro0080 tribute to the personal involvement and sustained effort of Dr Mohamed Ghoneim. Why does his system work so well? A major reason is $\mathrm{Dr}$ Ghoneim's personal commitment to its success. He recruited computer scientists from the University of Cairo, engineers from General Electric and members of his own faculty to collaborate and build a system that works as well for the doctor in the clinic and the surgeon in the operating room as it does for the professor or statistician in the office.

The key principles that Dr Ghoneim insisted on from the beginning were full integration, rapid response and simplicity. For the doctor, the system had to integrate all the information needed to practice medicine: the demographic data, medical history and observations made during physical examinations, laboratory, radiology and pathology reports, as well as images of the studies and photomicrographs of specimens. The system had to appear seamless to the physician: no more than two clicks of the mouse to move between systems; for example, from the medical history to a CT image. Most of the clinical findings can be entered using drop-down menus with standardized dictionaries, but physicians may still type free text responses in some fields. To prevent delays, the system response time is rapid.

For a system to be widely accepted by practicing physicians, it must be fully integrated into the clinical workflow. Today, there are few, if any, acceptable systems that can be purchased off the shelf. A truly satisfactory system will require the kind of dedicated, intelligent, persistent demands that $\mathrm{Dr}$ Ghoneim placed on his information technology group; refusing to accept anything less than a system that would deliver the right information in the right way at the right time. He has clearly demonstrated that it can be done. 